\title{
Féeries
}

Études sur le conte merveilleux, XVII ${ }^{\mathrm{e}} \mathrm{XIX} \mathrm{X}^{\mathrm{e}}$ siècle

16 | 2020

Le conte, les mythes antiques, la Bible

\section{D’Éros à la Bête : la chambre des secrets}

From Eros to the Beast: the chamber of secrets

\section{Gaëlle Le Guern-Camara}

\section{(2) OpenEdition}

Journals

Édition électronique

URL : https://journals.openedition.org/feeries/2842

DOI : $10.4000 /$ feeries. 2842

ISSN : 1957-7753

\section{Éditeur}

UGA Éditions/Université Grenoble Alpes

\section{Édition imprimée}

ISBN : 978-2-37747-194-2

ISSN : 1766-2842

\section{Référence électronique}

Gaëlle Le Guern-Camara, « D'Éros à la Bête : la chambre des secrets », Féeries [En ligne], 16 | 2020, mis en ligne le 07 janvier 2021, consulté le 10 décembre 2021. URL : http://journals.openedition.org/ feeries/2842 ; DOI : https://doi.org/10.4000/feeries.2842

Ce document a été généré automatiquement le 10 décembre 2021.

(c) Féeries 


\title{
D’Éros à la Bête : la chambre des secrets
}

\author{
From Eros to the Beast: the chamber of secrets
}

\author{
Gaëlle Le Guern-Camara
}

1 L'histoire de "Psyché et Cupidon » fait partie des mythes fondateurs dans la culture occidentale. Le premier récit littéraire connu qui nous y donne accès date du II $^{\mathrm{e}}$ siècle : il est inséré dans Les Métamorphoses ou l'Âne d'or d'Apulée ${ }^{1}$. Sa fortune philosophique et littéraire est immense : les interprétations et les adaptations sont innombrables. Dans la préface de l'édition de 1695 de ses trois récits en vers, « Grisélidis », « Peau d'Âne » et "Les Souhaits ridicules», Charles Perrault l'assimile à un "conte de Vieille», s'appuyant pour cela sur la situation d'énonciation : l'histoire est contée par une vieille femme à une jeune fille captive pour la distraire ${ }^{2}$. Il rapproche ainsi ce récit des contes issus de la tradition orale, et semble écarter les spécificités liées à sa dimension mythologique.

2 Or, depuis le xviI ${ }^{\mathrm{e}}$ siècle, on trouve une trame narrative très proche de celle de l'histoire de «Psyché et Cupidon» dans des contes littéraires : il s'agit tout d'abord de plusieurs récits de l'italien Giambattista Basile, évoquant des traditions orales populaires, parus dans Le Conte des contes entre 1634 et $1636^{3}$; puis en 1740, Madame de Villeneuve publie anonymement le récit «La Belle et la Bête", qui sera repris et rendu célèbre par la version de Madame Leprince de Beaumont en 1756; au XIx ${ }^{e}$ siècle, les Grimm tout comme Alexandre Afanassiev écriront des récits semblant puiser aux mêmes sources ${ }^{4}$. Aux xix ${ }^{e}$ et $\mathrm{Xx}^{\mathrm{e}}$ siècles, au moment des collectes des folkloristes et des ethnologues en vue de fixer le patrimoine français des traditions orales, des contes populaires présentant d'indéniables ressemblances avec ces contes littéraires sont recueillis. La structure de ces textes montre de forts liens de parenté, au point qu'ils ont été associés au début du xxe siècle à un même conte-type, "À La Recherche de l'époux disparu » (ATU 425). Le folkloriste suédois Jan-Öjvind Swahn les rassemble dans une monographie intitulé The Tale of Cupid and Psyche en $1955^{5}$. Ses analyses structurales sont reprises dans Le Catalogue du conte populaire français par Paul Delarue et Marie- 
Louise Ténèze ${ }^{6}$, montrant les liens entre le récit d'Apulée, les versions littéraires du $\mathrm{XVIII}^{\mathrm{e}}$ siècle et des versions orales collectées ultérieurement.

On s'interrogera sur la légitimité à associer deux traditions : celle issue de l'héritage mythologique gréco-romain, autour des figures de "Psyché et Cupidon», et celle de contes adoptant une même structure narrative mais autour de personnages très différents, correspondant au type générique de «La Belle et la Bête ». Pour cela, nous verrons dans un premier temps la façon dont le récit de "Psyché et Cupidon » s'est constitué ; puis nous analyserons quelques exemples de chemins parallèles pris dans des textes autour des figures de «La Belle et la Bête»; enfin nous confronterons les deux traditions pour tenter de mettre en lumière la nature des liens qui les unissent.

\section{Retour sur l'histoire d'un mythe}

Dans son ouvrage L'Invention d'un mythe: Psyché. Allégorie et fiction, du siècle de Platon au temps de La Fontaine, Véronique Gély retrace précisément la genèse du mythe de Psyché et Cupidon ${ }^{7}$ : si le premier récit littéraire connu est celui d'Apulée, les personnages sont des figures allégoriques célèbres depuis la Grèce antique, associées à la philosophie platonicienne. Elles ont été sources d'inspiration pour de nombreux artistes. L'originalité d'Apulée a été d'en faire un conte, qui constitue une parenthèse à l'intérieur de son récit Les Métamorphoses ou l'Âne d'or. Pour cela, on peut supposer qu'il s'est inspiré, comme le feront Basile, Straparole ou Perrault bien des siècles plus tard, de sources orales populaires. L'originalité de son récit consiste à façonner un texte littéraire avec des figures mythologiques qu'il insère dans une trame narrative dans laquelle on ne peut démêler précisément ce qui relève de l'invention de l'artiste ou de la transmission orale. La conséquence est la création d'un mythe littéraire : celui de Psyché, mortelle d'une grande beauté, dont le nom signifie "Âme ». Cupidon, le dieu Amour, tombe passionnément amoureux d'elle, en dépit de la féroce jalousie de sa mère, la déesse Vénus. Ce mythe peut se prêter à trois types de lecture: une lecture allégorique, dans le prolongement des interprétations platoniciennes qui seront ensuite récupérées par les exégètes chrétiens; une lecture existentielle, dans laquelle Psyché fait figure de modèle dans sa trajectoire initiatique ; une lecture esthétique, qui privilégie la beauté un peu mystérieuse du récit et de sa mise en forme. Ces différentes lectures connaissent des succès variables au fil des siècles : l'interprétation de l'œuvre évolue d'une époque à l'autre.

5 Ainsi, en 1669, Les Amours de Psyché et Cupidon de Jean de La Fontaine constitue un tournant dans l'histoire du mythe. Cette œuvre met particulièrement en avant la dimension esthétique. Jean Rousset l'analyse ainsi :

Avec l'air de la plus grande nonchalance, Psyché est une œuvre centrée sur d'autres œuvres, elles-mêmes issues d'œuvres antérieures, qui s'y reflètent à l'infini comme dans une galerie des glaces où se rassemblerait tout un musée, où s'édifierait un système des beaux-arts ${ }^{8}$.

6 Tout, depuis la forme choisie qui encadre le récit par un débat littéraire entre quatre amis, jusqu'au décor choisi pour ce débat, qui est le château de Versailles et ses jardins encore en pleine élaboration, concourt à faire de cette œuvre une réflexion sur l'art. Mais parallèlement, le fabuliste qu'est Jean de La Fontaine ne peut négliger de donner à son récit une dimension morale, que Marcel Raymond résume en ces termes : 
La leçon que formule La Fontaine intéresse la psychologie humaine. C'est une morale, et moins courte qu'il ne semble : «[...] tenez-vous certaine que du moment que vous n'aurez plus rien à souhaiter, vous vous ennuierez. [...] l'entière satisfaction et le dégoût se tiennent la main! [...] le meilleur pour vous est l'incertitude, et qu'après la possession, vous ayez toujours de quoi désirer : c'est un secret dont on ne s'était pas encore avisé. » Le bien consiste à demeurer toujours en deçà du point où commencera, infailliblement, la chute, le regret, où la musique ne sera plus aussi suave que tout à l'heure'.

7 Cette morale est très éloignée des interprétations philosophiques et théologiques complexes auxquelles ont donné lieu les lectures allégoriques. La dimension mythologique donne au récit son esthétique propre, mais la réflexion proposée est universelle. Molière et Corneille procèderont de même dans leur tragédie-ballet intitulée Psyché, qui sera jouée deux ans plus tard: ils gardent le cadre mythologique qui séduit le public de la Cour, mais ils étoffent la psychologie des personnages, par des dialogues qui les humanisent au détriment de leur dimension allégorique. Ainsi, on voit d'abord Psyché succomber à l'émerveillement de sa nouvelle demeure, avant de manifester de la tristesse face à une situation dont l'apparente perfection ne suffit finalement pas à la contenter. Le personnage de l'Amour s'en fait l'écho, dans cette réplique :

Mais d'où vient qu'un triste nuage

Semble offusquer l'éclat de ces beaux yeux?

Vous manque-t-il quelque chose en ces lieux?

Des vœux qu'on vous y rend dédaignez-vous l'hommage ${ }^{10}$ ?

On retrouve la morale de La Fontaine dans cette pièce qui apparaît comme une sorte d'adaptation théâtrale du récit qui l'a précédé.

Pourtant, quand Perrault aborde à son tour la question du message contenu dans l'histoire de "Psyché et Cupidon ", il fait table rase à la fois des traditions anciennes et des relectures de son temps, pour aboutir à une vision du mythe qui lui dénie toute possibilité de transmettre une morale :

À l'égard de la Morale cachée dans la Fable de Psyché, Fable en elle-même très agréable et très ingénieuse, je la comparerai avec celle de Peau d'Âne quand je la saurai, mais jusqu'ici je n'ai pu la deviner. Je sais bien que Psyché signifie l'Âme ; mais je ne comprends point ce qu'il faut entendre par l'Amour qui est amoureux de Psyché, c'est-à-dire de l'Âme, et encore moins ce qu'on ajoute, que Psyché devait être heureuse, tant qu'elle ne connaîtrait point celui dont elle était aimée, qui était l'Amour, mais qu'elle serait très malheureuse dès le moment qu'elle viendrait à le connaître : voilà pour moi une énigme impénétrable. Tout ce que l'on peut dire, c'est que cette Fable de même que la plupart de celles qui nous restent des Anciens n'ont été faites que pour plaire sans égard aux bonnes mœurs qu'ils négligeaient beaucoup. Il n'en est pas de même des contes que nos aïeux ont inventés pour leurs Enfants. Ils ne les ont pas contés avec l'élégance et les agréments dont les Grecs et les Romains ont orné leurs Fables; mais ils ont toujours eu un très grand soin que leurs contes renfermassent une moralité louable et instructive. Partout la vertu y est récompensée, et partout le vice y est puni ${ }^{11}$.

Dans ce passage de sa préface, Perrault établit une équivalence intéressante entre le mythe de Psyché et Cupidon et les contes de tradition orale. Néanmoins, à l'encontre de La Fontaine et de Molière, il dénie au mythe toute possibilité de délivrer un message d'ordre moral. Il faut replacer son propos dans le contexte de la Querelle des Anciens et des Modernes, Perrault ayant occupé une place prépondérante dans le camp des Modernes. Véronique Gély montre le tour de force réalisé par Perrault : 
Avec habileté, Perrault a donc réussi à aller contre les préjugés des Anciens, à hisser Peau d'Âne au rang de véritable conte latin. Mais cette apparente concession aux Anciens n'est évidemment qu'une feinte : il n'est pas question pour Perrault d'en rabattre sur la supériorité des Modernes. Aussi continue-t-il en renversant le rapport entre les deux contes, l'ancien et le moderne : ayant réussi à faire admettre que Psyché est un conte, il continue en démontrant que, en tant que conte, elle est inférieure à Peau d'Âne. Pourquoi? Parce que le seul critère d'excellence d'une œuvre littéraire est pour lui sa valeur morale. Or Psyché n'a pas de valeur morale, à la différence de Peau d'Âne.

11 Or, si le parallèle établi par Perrault entre le récit de Psyché et Cupidon et les contes de tradition orale est pertinent, il importe de revenir sur la distinction qu'il établit à propos de la morale: les contes populaires seraient-ils, plus que la mythologie, empreints de moralité ? Perrault fonde son assertion sur une certaine pratique des contes merveilleux: il évoque des situations où, face à un destinataire enfant, le conteur donnait à son récit une dimension éducative. Il s'agit à présent de voir, à travers quelques exemples proches du mythe de "Psyché et Cupidon ", d'autres chemins empruntés par les contes, en nous appuyant notamment sur des versions issues de l'oralité.

\section{Les chemins de l'oralité}

12 Dans les cultures traditionnelles, les contes merveilleux, dont fait partie le conte-type ATU 425, étaient destinés à tout le groupe social. L'image du "conte de Vieille » diffusée par Perrault provient des modalités particulières qui ont permis aux lettrés de son époque d'avoir accès à cette culture : il s'agissait des nourrices qui, issues des milieux populaires, racontaient ces histoires aux enfants dont elles avaient la charge. C'est ainsi que, petit à petit, la dimension éducative a prévalu dans la transmission des contes, et que les écrivains se la sont réappropriée. Dans les traditions populaires, les contes merveilleux se transmettaient oralement lors des veillées, et étaient avant tout des moments de plaisir partagé. Si les contes évoluaient nécessairement au fil du temps, déformés par le bouche-à-oreille et par les inévitables modifications liées aux évolutions des sociétés, ils gardaient néanmoins une stabilité remarquable au niveau de leur trame et de leurs motifs.

13 Concernant les traditions orales autour $\mathrm{du}$ conte-type ATU 425, proches structurellement du mythe de "Psyché et Cupidon », parmi les 122 versions recensées par Le Catalogue du conte populaire français de Paul Delarue et Marie-Louise Ténèze ${ }^{12}$, nous avons choisi de nous appuyer particulièrement sur les textes suivants: "L'Homme-Crapaud » et "Le Loup gris », recueillis en Bretagne par François-Marie Luzel en $1863^{13}$; «Le Roi des Corbeaux », recueilli en Gascogne par Jean-François Bladé à la fin du XIX ${ }^{\mathrm{e}}$ siècle ${ }^{14}$; «Le Crapaud », recueilli en Bretagne par Geneviève Massignon en $1953^{15}$; «Le Conte du Crapaud », recueilli en Auvergne par Henri Pourrat autour de 1950 , et ayant donné lieu à une réappropriation littéraire ${ }^{16}$. L'ensemble des versions recueillies marque une très grande stabilité des motifs, parmi lesquels on relève en particulier le fait qu'une jeune fille s'unit à un être marginal qui est dans la majorité des cas un époux-animal. En cela, ces versions issues de l'oralité se distinguent nettement du récit mythologique de "Psyché et Cupidon ", avec la figure de l'épouxdivin. En revanche, elles s'inscrivent dans la continuité des contes littéraires de Giambattista Basile, Madame de Villeneuve, Madame Leprince de Beaumont, Jacob et 
Wilhelm Grimm et Alexandre Afanassiev, mais en insistant davantage que ces derniers sur l'animalité de l'époux.

D'une version à l'autre, l'animal peut être un loup, un bouc, un crapaud, un serpent, un corbeau ou un porc, peu importe: dans tous les cas, l'animalité s'accompagne d'un dégoût qui devra être surmonté. «L'Homme-Crapaud » montre ainsi l'apparition de la Bête au père de l'héroöne: "Au moment où il était penché sur l'eau, emplissant sa cruche, un crapaud lui sauta à la figure et s'y colla si bien que tous ses efforts pour l'arracher demeurèrent inutiles ${ }^{17}$. » Dans «Le Loup gris ", issu de la même collecte, on la présente en ces termes : «Un jour, qu'il allait conduire ses vaches au pâturage, il rencontra un grand loup gris (de vieillesse sans doute), qui vint tout droit à lui et lui demanda une de ses filles en mariage ${ }^{18}$. $\gg$ Certes, l'animal connaîtra une métamorphose physique qui le rendra aussi beau que le dieu Amour, mais la présentation liminaire donne des personnages des images qui semblent incompatibles. Il est vraisemblable que ces traditions orales gardent la trace de rituels très anciens, dans lesquels la phase d'animalisation correspondait à une étape initiatique. Claude Lecouteux, pour sa part, fait référence à la question du Double animal, qu'il définit en ces termes :

Dans les mentalités païennes, donc, l'homme possède un Double apte à changer d'aspect en se détachant du corps, soit pendant le sommeil ou la transe, soit à volonté, comme c'est le cas des magicien(ne)s et des sorcier(e)s. Il est donc tout à fait possible qu'autrui puisse, par les moyens appropriés relevant de la connaissance des choses occultes, science d'un ordre particulier et interprétée en bonne et mauvaise part, vous métamorphoser, de même qu'il vous est possible de changer autrui en bête. Là où les choses se compliquent, c'est que la christianisation, dont saint Augustin fournit un bon exemple, a effacé et acculturé cette croyance dans la plupart des pays de l'Occident médiéval, sauf dans le domaine germanique. Les narrateurs ne savent plus qu'un coup de baguette magique ou qu'une incantation agit sur l'autre moi, le libère, c'est-à-dire l'affranchit des liens du corps, et peut le condamner à l'errance sous la forme d'un animal $^{19}$.

Cette croyance au Double animal est à l'origine des légendes de loups-garous au Moyen Âge, dont certaines sont rapportées dans l'anthologie de Claude Lecouteux intitulée Elle courait le garou ${ }^{20}$. Elle imprègne également de nombreux contes, dans lesquels l'aspect rituel se perd progressivement au profit d'une quête personnelle, qui garde néanmoins une dimension initiatique. Mais l'initiation est en quelque sorte christianisée (en tout cas pour les versions auxquelles on a accès aujourd'hui, qui sont issues pour la plupart de collectes ayant été réalisées au $\mathrm{xIX}^{\mathrm{e}}$ et $\mathrm{xx}^{\mathrm{e}}$ siècles). L'animalité devient surtout symbolique, mettant en jeu une opposition entre nature et culture.

Dans le corpus de contes qui nous intéresse, ce qui est primordial, ce n'est pas tant la trajectoire d'un individu que celle d'un couple. En effet, les deux protagonistes vont se réaliser l'un à travers l'autre: tout d'abord, l'homme, devenu bête suite à une malédiction, ne peut retrouver son humanité que grâce à un amour sincère. Dans «Le Crapaud ", le héros dit à sa jeune épouse, qui a su l'aimer en dépassant les apparences : "J'ai été transformé en ce bel homme que tu vois, car j'étais en punition sous la forme d'un crapaud, et maintenant, grâce à toi, je suis délivrée ${ }^{21}$. Mais de même que l'homme évolue grâce à sa femme, cette dernière se métamorphose au contact de la bête : elle apprend à l'aimer sous sa forme animale. Pour cela, elle dépasse ses préjugés. Les personnages des sœurs ont d'ailleurs pour principale fonction de mettre en valeur l'opposition entre celles qui restent prisonnières des stéréotypes et de la rigidité dans laquelle la société les a enfermées et celle qui est capable de s'en affranchir. Par 
exemple, dans «Le Conte du Crapaud " d'Henri Pourrat, les sœurs réagissent ainsi à l'annonce du mariage de l'héroïne : «Un crapaud, quelle horreur ! eh bien! c'est pour le coup !...Coucher avec cette dégoûtation...De la vie des vivants! Tu n'iras pas, je pense $^{22}$ ? » De l'héroïne, en revanche, on apprend : «Car elle, elle n'avait aucun dégoût pour aucune créature; et ses manières savaient gagner le cœur de tout ce qui l'approchait ${ }^{23}$.»

Mais ce qui semble au départ une prédisposition à la tolérance et à l'abnégation (dans la plupart des versions, l'héroïne accepte le mariage avec la bête pour sauver son père) prend rapidement, au contact de l'époux-animal, une autre tournure. On le voit par exemple dans «L'Homme-Crapaud»: «Les deux sœurs de la mariée venaient parfois lui faire visite, et elles étaient bien étonnées de la trouver si gaie; elle chantait et riait continuellement ${ }^{24}$. » L'héroïne est métamorphosée au contact de l'époux. Certes, la vie commune avec lui a révélé un secret qui semble relativiser l'importance de sa part animale, comme le découvrent ses sœurs en l'espionnant:

Une nuit, elles vinrent, tout doucement, regarder par le trou de la serrure, et elles furent bien étonnées de voir un prince jeune et beau, au lieu d'un crapaud !

- Tiens ! tiens ! le beau prince !... Si j'avais su !... disaient-elles alors.

Elles entendirent alors le prince dire ces paroles à sa femme :

- Demain, je dois aller en voyage, et je laisserai à la maison ma peau de crapaud.

Veillez bien à ce qu'il ne lui arrive pas de mal, car j'ai encore un an et un jour à rester sous cette forme ${ }^{25}$.

18 La bête est en fait un beau prince la nuit, ce qui permet d'expliquer le bonheur que manifeste l'héroïne dans sa vie de jeune épouse. Néanmoins, la part animale est loin d'avoir totalement disparu : la peau de bête doit être soigneusement conservée après la métamorphose, sous peine de mettre en péril l'harmonie du couple. On le voit bien dans « Le Crapaud»:

Peu après les noces le Crapaud a décidé de partir en voyage avec sa femme ; celle-ci s'est tellement dépêchée qu'elle est partie en oubliant la clé de l'armoire où était conservée la peau de crapaud. À quelques jours de là, ses sœurs veulent ouvrir cette armoire, et qu'est-ce qu'elles voient ? un sac. Elles l'ouvrent et trouvent la serviette blanche, et dedans la peau de crapaud.

- Comment? Tiens, voilà sa peau!

- Qu'allons-nous en faire ? On ne va pas garder ça dans cette armoire.

Ce jour-là elles étaient justement en train de boulanger : le four à pain était chaud ;

- Jette donc cette vieille peau dans le four, à brûler !

Aussitôt que la peau a été brûlée, le Crapaud qui était bien loin avec sa femme s'est mis à lui dire :

- Ah! voilà que le malheur est arrivé.

- Qu'est-ce qu'il y a ?

- Ne t'avais-je pas dit de veiller sur ma peau de crapaud ? Il ne fallait surtout pas la laisser détruire. Et maintenant, elle a été brûlée dans le four à pain de tes sœurs. Nous ne pouvons plus rester ensemble tous les deux. Désormais, il faut que je te quitte ${ }^{26}$.

19 Quand la peau animale est détruite, les deux époux sont contraints de se séparer. Même si elle est temporaire et conduit à une autre phase de l'initiation (la femme doit reconquérir le mari, qu'elle cherche en usant des souliers de fer, et le sortir de l'amnésie alors qu'il est sur le point de se marier avec une seconde épouse), cette rupture marque l'importance de l'animalité dans la relation entre les deux protagonistes. Il s'agit à première vue d'une métaphore pour exprimer la sensualité au sein du couple. Pierre Péju suggère quant à lui une autre piste: « On comprend que le 
désir, tout le désir ne peut tenir dans le cadre étroit des relations affectives humanisées, socialisées. Le désir s'adonne, on l'a vu, aux métamorphoses, parcourt les états animaux, végétaux ${ }^{27}$. $"$ Ce qui se jouerait alors dans le récit, ce serait l'infini du désir, par opposition au cadre très restreint auquel le réduisent parfois les sociétés. Les pulsions animales, associées à la notion de bestialité, qui renvoie au vice et à la saleté, sont ici revalorisées. L'héroïne renoue grâce à la bête avec une part instinctive d'ellemême qui avait été étouffée. La bête, quant à elle, s'humanise au contact de celle qu'il a choisi d'aimer.

Ce mariage échappe au contrôle social auquel sont d'habitude soumises les unions : il y a dans ce couple une exogamie maximale, qui excède ce que les sociétés sont dans la plupart du temps capables de tolérer. Il est ici question, avant toute chose, d'une altérité qui vise à se métamorphoser en complémentarité. La quête est individuelle, elle prend le pas sur le message social du conte, même si la dernière partie de l'histoire vise dans la plupart des cas à marquer le retour du couple à une union socialisée, qui a seule vocation à devenir pérenne. On le voit dans « Le Conte du Crapaud » d'Henri Pourrat :

Le conte ne raconte pas ce que devinrent les deux aînées, la paresseuse et la méchante : elles crevèrent bien de dépit, si elles voulurent. Mais la jeune, la belle et la gentille, ce beau monsieur la mit dans une tour. Puis le moment venu, il l'épousa en grande fête. Et le soir même, elle et son père le roi, il les conduisit dans son château des magnificences où tout était marqué au nom de la belle ${ }^{28}$.

Néanmoins, certaines versions laissent planer un doute, donnant l'impression que la marginalisation du couple, au fond des bois, pourrait éternellement durer. C'est le cas dans la clôture du «Loup gris »: "Et aussitôt ils se levèrent de table tous les deux et partirent, au milieu du silence et de l'étonnement général. Ils revinrent à leur ancien château, où ils retrouvèrent leur enfant, et vécurent heureux, je présume, car depuis, je n'ai pas entendu parler d'eux ${ }^{29}$.» Cet amour caché au fond des bois, marqué par l'hybridité, est à mille lieues des morales éducatives des « contes de Vieille » dont nous parlait Perrault. Pourtant, il est bien issu d'une tradition héritée de "nos aïeux ", entrée en littérature avec les œuvres de Basile, Madame de Villeneuve et Madame Leprince de Beaumont, et diffusée en parallèle par le biais de l'oralité, avec d'incessants allers-retours entre ces deux vecteurs de transmission. Il importe à présent de confronter les contes que nous avons convoqués dans notre analyse avec le récit mythologique de Psyché et Cupidon, afin de déterminer quels liens on peut dégager entre ces deux pans de notre héritage culturel autour d'un même type de récit.

\section{Du dieu à la bête}

Le mythe de "Psyché et Cupidon », en dépit des ressemblances structurelles, possède une singularité qui semble le distinguer radicalement de l'ensemble des autres récits associés au conte-type ATU 425, «À La Recherche de l'époux disparu » : Cupidon, dieu de l'Amour, semble en effet fonctionner comme une parfaite antithèse à la mystérieuse Bête rendue célèbre par Madame Leprince de Beaumont. En dépit d'une trame commune, quels liens peut-on établir entre des récits présentant un tel écart dans la construction d'un des personnages principaux? Dans le mythe, le statut divin du personnage masculin qui devient son époux élève l'héroïne au point que Jupiter luimême lui accorde l'immortalité, alors que dans les contes l'épouse de la bête apparaît comme marginalisée, dans un premier temps du moins. Et pourtant, à bien des égards, 
la bête se rapproche du dieu. Cela se manifeste tout d'abord par son lieu de vie. Ainsi, chez Apulée, la demeure de Cupidon est ainsi décrite :

Dès qu'on était dans l'entrée on comprenait qu'on avait sous les yeux la lumineuse et luxueuse garçonnière d'un dieu. Des piliers d'or soutenaient des plafonds à caissons soigneusement incrustés d'ivoire et de thuya, tous les murs étaient plaqués d'argent ciselé en relief et de bêtes genre bête de cirque et autres, qui couraient à la figure du visiteur ${ }^{30}$.

Ce qui frappe en premier, c'est le luxe qui se dégage de ce décor doré et argenté. Mais la présence des motifs animaux est également intéressante à relever. Dans «Le Roi des Corbeaux », le narrateur plante le décor suivant :

Les Corbeaux partirent, et la reine entra dans son château. Il était sept fois plus grand que l'Église de Saint-Gervais de Lectoure. Partout brûlaient des lumières. Les cheminées flambaient, comme des fours de tuiliers. Pourtant, la reine ne vit personne. Tout en se promenant de chambre en chambre, elle arriva dans une grande salle, où il $\mathrm{y}$ avait une table, chargée de plats et de vins de toute espèce. Un seul couvert était mis. La reine s'assit. Mais elle n'avait pas le cœur à boire et à manger, car elle pensait toujours aux siens et à son pays. Une heure après, la reine s'alla coucher dans un lit, fermé de rideaux d'or et d'argent, et attendit, sans dormir, en laissant brûler la lumière ${ }^{31}$.

On retrouve ici la même magnificence que dans la demeure de Cupidon, caractérisée par l'argenté et le doré, la lumière. Ici la référence à l'animalité renvoie non plus à des éléments du décor, mais désigne directement l'époux. Néanmoins, un lien semble ainsi s'établir entre les deux personnages. On voit l'ambiguïté qui réside à la fois dans la figure du dieu associé à un animal par les œuvres d'art qui l'entourent, et dans la figure de l'époux-animal qui possède une demeure digne d'un dieu. La complexité du personnage de Cupidon est d'ailleurs annoncée d'entrée de jeu chez Apulée, dans un portrait qui le désacralise au moment où Vénus requiert son intervention :

Et de suite elle appelle son garçon, l'écervelé volant, le garnement polissonnant sans souci des bonnes mœurs, le galopin qui court la nuit bardé de flèches et de brandons pour aller chez les autres semer la zizanie dans leurs ménages, le galapiat aux mille scandales impunis, le bon à rien qui vaille ${ }^{32}$.

Cette image d'enfant farceur, dépourvu de morale, n'est pas sans lien avec celle de l'époux-animal associé à un monstre. D'ailleurs, cette vision de Cupidon en être monstrueux, ne connaissant aucune limite, est celle qui apparaît dans l'oracle chez La Fontaine :

L'époux que les Destins gardent à votre fille Est un monstre cruel qui déchire les cœurs, Qui trouble maint État, détruit mainte famille Se nourrit de soupirs, se baigne dans les pleurs. À l'univers entier il déclare la guerre, Courant de bout en bout un flambeau dans la main : On le craint dans les cieux, on le craint sur la terre; Le Styx n'a pu borner son pouvoir souverain ${ }^{33}$.

L'oracle, dans une certaine mesure, dit vrai : Cupidon n'est-il pas en effet un monstre, bras armé de Vénus? Il est semblable à la Bête du conte traditionnel qui détruit les familles, et tout comme elle c'est le véritable amour, réciproque, qui lui permettra de changer. Dans le récit d'Apulée et ceux de ses successeurs, la dimension mythologique n'exclut pas la double initiation des protagonistes : même si c'est Psyché qui rejoint le monde des dieux, tandis que Cupidon retrouve l'olympe qu'il avait quitté pour la séduire, l'époux lui aussi subit une mutation, et accomplit une révolution qui pour être 
intérieure n'en est pas moins réelle. Il s'humanise par sa relation avec Psyché. On voit donc l'écart entre le dieu et la bête se réduire, même s'il ne disparaît pas tout à fait.

S'il fallait déterminer un motif qui permette de donner une signification commune à l'ensemble des récits, en dépit des écarts, ce serait sûrement celui de l'altérité dans la relation amoureuse. Mais cette question est corrélée à une autre : celle du secret. En effet, que le héros soit bête ou dieu, il est l'Éros, le désir amoureux, qui ne se révèle qu'au cœur de la nuit, dans une intimité nimbée de mystère. Que l'héroïne connaisse ou non l'identité de l'époux, elle doit respecter son mystère. Il suffit d'une goutte de cire qui coule sur le corps parfait de l'aimé, révélant l'indiscrétion de la jeune femme, ou de l'irruption des sœurs jalouses dans le sanctuaire conjugal pour brûler la peau de la bête, et c'est toute la relation qui est remise en jeu. Dans sa retraite, entre l'ombre et la lumière, le couple essaie de se construire à l'abri des regards d'autrui. Mais la "faute " de l'héroïne n'est en fait qu'une étape nécessaire dans l'élaboration de la relation : le couple ne peut éternellement vivre au fond des bois ou d'une grotte, la parenthèse enchantée ne peut éternellement durer. La question de la relation au groupe social, forcément, se pose. La violence de la rupture appelle une reconquête : il faut trouver le fragile équilibre qui permettra de concilier couple et société, respect du groupe et de l'intimité, vaste monde et chambre des secrets.

Ainsi, le mythe de "Psyché et Cupidon » et les contes mettant en scène les personnages du type de «La Belle et la Bête » constituent deux facettes de la même histoire. Si le mythe élaboré à partir du récit d'Apulée a donné lieu à de multiples exégèses, il peut aussi se lire comme un conte dont les enjeux se rapprochent fortement d'autres récits dépourvus de toutes références mythologiques. Les motifs varient, semblent parfois s'opposer, mais le fond du récit reste le même: il questionne le couple, dans son intimité et au sein de la société. Si «Psyché et Cupidon», «Le Loup gris » ou «Le Roi des Corbeaux " sont des réalisations artistiques singulières, elles déroulent des itinéraires de vie similaires. Si elles mettent en lumière le fonctionnement psychique des héros, selon la thèse de Bruno Bettelheim ${ }^{34}$, elles dévoilent également la façon dont les interactions sociales contribuent à les façonner (c'est ce que montre François Flahault dans La Pensée des contes ${ }^{35}$ ) : entre liens de sang et liens d'alliance, héros et héroïnes du conte-type ATU 425 tracent leur route, cherchent leur place, et construisent petit à petit leur identité.

\section{NOTES}

1. Apulée, Les Métamorphoses ou l'Âne d'or, trad. O. Sers, Paris, Les Belles Lettres, coll. «Classiques en poche ", 2007.

2. C. Perrault, Contes, Paris, Gallimard, coll. «Folio Classique », 1981, p. 52.

3. G. Basile, Le Conte des contes, ou le divertissement des petits enfants, trad. F. Decroisette, Paris, Circé, 1995. On peut citer parmi ces textes «Le Verrou» (p. 200-203), «La Merveille» (p. 412-417), « L'Arbre d'or » (p. 418-426), ainsi que le récit-cadre.

4. J. et W. Grimm, Contes pour les enfants et la maison, trad. N. Rimasson-Fertin, Paris, Corti, coll. «Merveilleux », 2009, t. 2. Il s'agit de «L'Alouette qui chante et sautille» (p. 12-18) et de «Le 
Poêle de fonte» (p. 205-210). A. Afanassiev, Contes populaires russes, trad. L. Gruel-Apert, Paris, Imago, 2009, t. 2. Il s'agit des deux récits intitulés «La Plume de Finiste le Faucon blanc» et «Finiste Clair Faucon II » (p. 332-343), ainsi que « Le Tsariévitch ensorcelé » (p. 98-100).

5. J. Ö. Swahn, The Tale of Cupid and Psyche (Aarne-Thompson, 425 et 428), Lund, CWK Glerup, 1955.

6. P. Delarue et M.-L. Ténèze, Catalogue du conte populaire français, Paris, Maisonneuve et Larose, 2002, p. 72-109.

7. V. Gély, L'Invention d'un mythe: Psyché. Allégorie et fiction, du siècle de Platon au temps de La Fontaine, Paris, Honoré Champion, coll. « Lumières classiques », 2006.

8. J. Rousset, «Psyché ou le génie de l'artificiel», dans P. Dandrey (dir.), La Fontaine. CEuvres "galantes». Le Songe de Vaux. Les Amours de Psyché et Cupidon, Paris, Klincksieck, coll. "Parcours critique », 1996, p. 182.

9. M. Raymond, « Psyché et l'art de La Fontaine », dans P. Dandrey (dir.), ouvr. cité, p. 160.

10. Molière, Euvres complètes, Paris, NRF Gallimard, coll. « Bibliothèque de La Pléiade », 2010, t. 2, p. 478.

11. C. Perrault, ouvr. cité, p. 53.

12. P. Delarue et M.-L. Ténèze, ouvr. cité.

13. F.-M. Luzel, Contes populaires de Basse-Bretagne, <http://fr.wikisource.org>.

14. J.-F. Bladé, «Le Roi des Corbeaux ", dans F. Raphoz (éd.), Des belles et des bêtes. Anthologie de fiancés animaux, Paris, Corti, coll. « Merveilleux », 2003, p. 214.

15. G. Massignon, Contes traditionnels des teilleurs de lin du Trégor (Basse-Bretagne), Paris, Éditions A. et J. Picard et Cie, 1965, p. 103-113.

16. H. Pourrat, Le Trésor des contes, Paris, Gallimard, coll. « Omnibus », 2009, t. 2, p. 1105-1110.

17. F.-M. Luzel, ouvr. cité.

18. Ibid.

19. C. Lecouteux, Fées, Sorcières et Loups-garous au Moyen Age, Paris, Imago, 1992, p. 127.

20. C. Lecouteux, Elle courait le garou, Paris, Corti, coll. « Merveilleux », 2008.

21. G. Massignon, ouvr. cité, p. 105.

22. H. Pourrat, ouvr. cité, p. 1110.

23. Ibid., p. 1109.

24. F.-M. Luzel, ouvr. cité.

25. Ibid.

26. G. Massignon, ouvr. cité, p. 106.

27. P. Péju, La Petite Fille dans la forêt des contes, Paris, Robert Laffont, 1997, p. 233.

28. H. Pourrat, ouvr. cité, p. 1110.

29. F.-M. Luzel, ouvr. cité.

30. "Iam scies ab introitu primo dei cuiuspiam luculentum et amoenum videre te diversorium. Nam summa laquearia citro et ebore curiose cauata subeunt aureae columnae, parietes omnes argenteo caelamine conteguntur bestiis et id genus pecudibus occurentibus ob os introeuntium. " (Apulée, ouvr. cité, p. 168-169).

31. J.-F. Bladé, ouvr. cité, p. 214.

32. "Et vocat confestim puerum suum pinnatum illum et satis temerarium, qui malis suis moribus contempta disciplina publica flammis et sagittis armatus per alienas domos nocte discurrens et omnium matronica corrumpens impune committit tanta flagitia et nihil prorsus boni facit. » (Apulée, ouvr. cité, p. 158-159).

33. J. de La Fontaine, Les Amours de Psyché et de Cupidon, Paris, GF Flammarion, 1990, p. 55.

34. B. Bettelheim, Psychanalyse des contes de fées, Paris, Robert Laffont, coll. « Pocket », 1976.

35. F. Flahault, La Pensée des contes, Paris, Anthropos, coll. « Psychanalyse », 2001. 


\section{RÉSUMÉS}

L'histoire de "Psyché et Cupidon » est un mythe fondateur de notre culture, dont la première trace littéraire se trouve dans Les Métamorphoses ou l'Âne d'or d'Apulée, et qui connaîtra de nombreuses réécritures. De nombreux contes racontent des histoires structurellement proches de ce mythe, mais avec une différence importante dans la construction des personnages : le héros n'est plus un dieu, mais une bête. C'est le cas notamment dans des contes littéraires comme « La Belle et La Bête » de Madame de Villeneuve ou de Madame de Leprince de Beaumont, ainsi que dans de nombreux textes issus de l'oralité. En dépit de leurs différences, mythe et contes se rejoignent en montrant des itinéraires de couples qui se construisent en surmontant leur altérité et en trouvant leur place au sein du groupe social.

The story of Psyche and Cupid is a founding myth of our culture. The first literary trace is in Les Métamorphoses ou l'Âne d'or by Apulée, which will knows many rewrites. Many tales tell stories structuraly close to myth, but with an important difference in the construction of the characters: the hero is no longer a god but a beast. It is true in literary tales as "Beauty and Beast" by Madame de Villeneuve or Madame Leprince de Beaumont, as well as many oral tradition tales. Despite their differences, myth and tales meet by showing itineraries of couples who are building by overcoming their otherness and finding their place in the social group.

\section{INDEX}

Keywords : Tale, myth, oral tradition, couple, society, animality, "Psyche and Cupid", "Beauty and Beast"

Mots-clés : Conte, mythe, tradition orale, couple, société, animalité, « Psyché et Cupidon », « La Belle et la Bête »

\section{AUTEUR}

\section{GAËLLE LE GUERN-CAMARA}

Université Paris-Diderot, Cerilac 